\title{
Outcome of appendicular or scapular osteosarcoma treated by limb amputation in cats: 67 cases (1997-2018)
}

Yuko Nakano, DVM, PhD ${ }^{1 *}$; Yumiko Kagawa, DVM, $\mathrm{PhD}^{2}$; Yumiko Shimoyama, DVM, $\mathrm{PhD}^{3}$; Tetsushi Yamagami, DVM, MS, PhD ${ }^{4}$; Kohji Nomura, DVM, MS ${ }^{4}$; Hidetaka Wakabayashi, MD, PhD ${ }^{5}$. Yoshifumi Sugiyama, MD, PhD ${ }^{6}$; Tetsuya Kobayashi, DVM, MSpVM ${ }^{1}$

\author{
JJapan Small Animal Cancer Center, Japan Small Animal Medical Center, Tokorozawa, Japan \\ ${ }^{2}$ North Lab, Hokkaido, Japan \\ 3IDEXX Laboratories, Tokyo, Japan \\ ${ }^{4}$ Veterinary Diagnostic Pathology Section, Marupi Lifetech Co, Ltd, Osaka, Japan \\ ${ }^{5}$ Department of Rehabilitation Medicine, Tokyo Women's Medical University Hospital, Tokyo, Japan \\ ${ }^{6}$ Division of Community Health and Primary Care, Center for Medical Education, The Jikei University School of Medicine, Tokyo, Japan \\ *Corresponding author: Dr. Nakano (yuko_watapoko@icloud.com)
}

\section{OBJECTIVE}

To evaluate the metastasis rate, survival time, and prognostic factors associated with appendicular or scapular osteosarcoma treated by limb amputation in cats.

\section{ANIMALS \\ 67 cats with histologically confirmed appendicular or scapular osteosarcoma treated by limb amputation.}

\section{PROCEDURES}

This retrospective cohort study included cats with histologically confirmed appendicular or scapular osteosarcoma between January 1997 and December 2018. A questionnaire survey was conducted at veterinary clinics where limb amputation was performed. Distant metastasis, local recurrence, and lymph node metastasis rates and survival time were determined. Factors associated with distant metastasis and survival were investigated.

\section{RESULTS}

The distant metastasis rate after limb amputation was $41.9 \%(26 / 62)$. The overall distant metastasis rate was $46.3 \%(31 / 67)$, including 5 cats with distant metastasis at the time of amputation. Osteosarcoma of the humerus resulted in distant metastasis in 6 of 7 cases. Osteosarcoma of the humerus was significantly associated with distant metastasis in univariate and multivariate analyses (adjusted OR, 9.56). The rate of lymph node metastasis after limb amputation was 3.0\% (2/66), and the local recurrence rate was $9.0 \%(6 / 67)$. The median survival time was 527 days. Age and tumor location were not significantly associated with survival time.

\section{CLINICAL RELEVANCE}

Distant metastasis occurs in approximately $40 \%$ of cats with appendicular or scapular osteosarcoma after limb amputation. In addition, osteosarcoma of the humerus has a particularly high incidence of distant metastasis. Detailed follow-up is therefore necessary, even after limb amputation, especially in cases of osteosarcoma of the humerus.

$\mathbf{F}$ line primary bone tumors are rare, with an incidence of $4.9 / 10,000$ cases. $^{1}$ Osteosarcoma is the most common primary bone tumor in cats, accounting for $70 \%$ to $80 \%$ of all primary bone tumors. ${ }^{2,3}$ Although osteosarcoma in cats usually occurs in relatively older cats ( 8 to 10 years of age), it has been diagnosed in cats with a wide range of ages ( 1 to 20 years of age). ${ }^{4-6}$ Osteosarcoma in cats is generally defined as tumors composed of malignant mesenchymal spindle cells that produce osteoid and is classified as osteosarcoma of medullary origin, parosteal osteosarcoma, fracture-associated osteosarcoma, and extraskeletal osteosarcoma. Osteosarcoma in cats of medullary origin occurs in appendicular or axial skeletal sites, with appendicular osteosarcoma being slightly more common than axial skeletal osteosarcoma. ${ }^{4,6,7}$ Appendicular osteosarcoma occurs more often in the hind limbs than forelimbs, and the distal femur, proximal tibia, humerus, and digits are the most commonly affected sites. ${ }^{6}$

Reports from the 1970 s to 2000 s indicated low metastasis rates of osteosarcoma in cats of $5 \%, 8 \%$, and $10 \%$, respectively. $3,5,6$ In 1974 , Liu et $\mathrm{al}^{2}$ reported that 4 of 5 cats with osteosarcoma treated by limb amputation survived for at least 26 months postoperatively, without recurrence or metastasis. In 1987 , Bietto et $\mathrm{al}^{4}$ reported that 12 cats with appendicular osteosarcoma underwent limb amputation and 
of the 11 available for follow-up, 6 remained alive at the time of the analysis, 13 to 64 months postoperatively. The median survival time of the other 5 cats that died was 49.2 months (range, 1 to 122 months). Only 1 of 11 cats had recurrence, and none developed metastasis. Furthermore, Kessler et al $^{6}$ reported that appendicular osteosarcoma in cats had a better prognosis after limb amputation, whereas recurrence was a common cause of euthanasia in cats with osteosarcoma arising in flat and irregular bones. Because of this low metastasis rate, osteosarcoma in cats has been considered to offer long-term survival following amputation alone..$^{4,6}$

However, metastasis of osteosarcoma in cats may not be rare. One study reported fracture-associated osteosarcoma with pulmonary metastasis 3 months after amputation. ${ }^{8}$ No large-scale studies have investigated the metastasis rate, prognosis, and associated factors in cats with appendicular or scapular osteosarcoma treated by limb amputation. We hypothesized that the metastasis rate of appendicular or scapular osteosarcoma in cats after amputation was higher than previously reported. Further information on the metastasis rate, prognosis, and associated factors may clarify the need for adjuvant chemotherapy and the importance of follow-up after surgery.

The aim of this study was to investigate the rate of metastasis and survival time of cats with appendicular or scapular osteosarcoma treated by limb amputation. We also explored the predictive factors related to metastasis and survival time.

\section{Materials and Methods}

\section{Design}

This retrospective cohort study was designed and reported in accordance with the Strengthening the Reporting of Observational Studies in Epidemiology (STROBE) guidelines. ${ }^{9}$ The study was exempt from approval by the Ethics Committee because it was a retrospective study based on reviews of medical records.

\section{Case selection}

We included cats with appendicular or scapular osteosarcoma that had been histologically confirmed at 3 pathology laboratories in Japan (Marupi Lifetech Co, Ltd, North Lab, and IDEXX Laboratories) between January 1997 and December 2018. Hind limb amputation in this study was defined as resection at the hip joint, resection with partial pelvis, or resection at the femur, and forelimb amputation was defined as resection at the shoulder joint, resection with the scapula, or resection at the humerus. Exclusion criteria were extraskeletal osteosarcoma and tumor lesions not confirmed in the bone. The number of cases during the study period determined the sample size.

\section{Outcome measurements}

The primary outcome was distant metastasis rate after limb amputation. The secondary outcomes were overall survival time, local recurrence rate, lymph node metastasis rate, and overall distant metastasis rate, including cats with distant metastasis detected at the time of amputation.

\section{Questionnaire methods and variables}

Information on breed, age, sex, and tumor location was obtained from the pathology request forms sent by the veterinarians at the veterinary clinics that performed the limb amputation to each pathology laboratory. Questionnaire-based surveys were conducted twice, in 2014 and 2020, respectively. Questionnaires were sent to the veterinary clinic where the limb amputation was performed to obtain information on the prognoses of the cases. Additional information obtained from written questionnaires to veterinarians included body weight; tumor location, the presence of lameness, radiographic abnormalities, and distant metastasis at the time of amputation; use of adjunctive treatments including surgery, radiation, chemotherapy, and NSAIDs; date of tumor recurrence, lymph node metastasis, or distant metastasis and its diagnosis method; and date and cause of death (tumor-related, other cause, or unclear; if the date of death was unclear, we recorded the last day the cat was confirmed to be alive).

Tumor location was classified as the scapula, humerus, radius or ulna, carpus, metacarpus or phalange of forelimb, femur, tibia or fibula, tarsus, metatarsus, or phalange of hind limb, based on the pathology request form, pathology report, and questionnaire. If the pathological examination revealed the location of the bone infiltrated by the tumor, the tumor location was determined from the pathology report; if the pathology report did not reveal the site of the tumor, the tumor location was determined from both the pathology request form and the questionnaire.

\section{Statistical analysis}

The local recurrence rate, distant metastasis rate, and lymph node metastasis rate were calculated with 95\% CIs. Cases in which the development of metastasis or recurrence was unknown according to the questionnaire were analyzed as no metastasis or no recurrence, to avoid overestimating the respective rates.

Overall survival was determined using KaplanMeier survival curves. Overall survival time was defined as the time from amputation to death from any cause. Cats that were lost to follow-up or remained alive were censored at the date of last contact. Cats that died but for which the date of death was unknown were also censored at the date of last contact.

Age and tumor location (humerus or other) were analyzed as variables to identify risk factors associated with distant metastasis and overall survival time. 
On the basis of a meta-analysis report ${ }^{10}$ in dogs in which humeral location was a negative prognostic factor for osteosarcoma in dogs in terms of survival time and disease-free interval, we evaluated the tumor location as humerus or other in our study. Cats were placed in 2 groups, below and above the median age, respectively. This analysis included both cases with distant metastasis detected at the time of limb amputation and cases with distant metastasis detected after limb amputation. The association with distant metastasis was determined by univariate analysis using the Fisher exact test and by multivariate analysis using a logistic regression model.

The association with overall survival time was evaluated by univariate analysis using the Log-rank test and multivariate analysis using the Cox proportional hazards model, with the assumption of proportional hazards tested graphically using the Schoenfeld residuals.

Values of $P<0.05$ were considered statistically significant. All analyses were performed using a statistical software package (Stata, version 13, Stata Corp LLC).

\section{Results}

A total of 102 cats were included in this study, of which questionnaires were collected from veterinarians for 74 cats (collection rate, $72.5 \%$ ). The medical records had been discarded for 7 of the 74 cats because the storage period for the medical records had expired, and no information was therefore available. Sixty-seven cats were therefore finally included in the descriptive and statistical analyses. The characteristics of the cats with osteosarcoma are shown (Table I), and tumor locations are depicted (Figure I). Pulmonary metastasis was evaluated by thoracic radiography at the time of limb amputation in 66 cats. Evaluations of local recurrence, lymph node metastasis, and distant metastasis during followup after limb amputation were unclear in 9, 40, and

Table I-Characteristics of 67 cats with osteosarcoma.

\begin{tabular}{lc} 
Characteristic & Value \\
\hline Median (IQR) age (y) & II $(8-13)$ \\
Sex (No. [\%]) & - \\
Intact males & $5(7.4)$ \\
Castrated males & $33(49.3)$ \\
Intact females & $6(9.0)$ \\
Spayed females & $23(34.3)$ \\
Median (IQR) body weight (kg) & $4.5(3.5-5.3)$ \\
Cat breed (No. [\%]) & - \\
$\quad$ Mixed breed & $53(79.1)$ \\
$\quad$ American Shorthair & $6(9.0)$ \\
Norwegian Forest Cat & $2(3.0)$ \\
Scottish Fold & $1(1.5)$ \\
$\quad$ Unknown & $5(7.4)$ \\
Lameness before surgery (No. [\%]) & $60 / 66(91)$ \\
Radiographic abnormalities before surgery & $64 / 65(98)$ \\
$\quad$ No. [\%]) & \\
\hline
\end{tabular}

— = Not applicable. IQR = Interquartile range.
11 cases, respectively. These cases were analyzed as no recurrence or no metastasis to avoid overestimating the respective rates.

Distant metastases at the time of surgery included pulmonary metastasis in 4 cases and bone metastasis in 1 case (5/67 [7.5\%]). Lymph node metastasis diagnosed by imaging at the time of surgery was observed in the axillary lymph node in 1 case (1/67 [1.5\%]). Six cats received chemotherapy after limb amputation, including carboplatin alone $(n=2)$, doxorubicin alone $(n=1)$, combination of carboplatin and doxorubicin $(n=1)$, and toceranib $(n=2)$.

The median follow-up time was 324 days (interquartile range [IQR], 151 to 566 days). Local recurrence occurred in 6 cats during follow-up (6/67 [9.0\%]; $95 \% \mathrm{CI}, 3.8 \%$ to $18.5 \%$ ), including 5 cases with an amputation site close to the tumor. Two cats with femur osteosarcoma with local recurrence had tumors located in the femoral head, 1 cat with osteosarcoma of the proximal humerus underwent amputation at the shoulder joint, 1 with osteosarcoma of the scapula had recurrence in the muscle at the site of resection, and 1 cat with osteosarcoma of the proximal tibia underwent amputation in the distal third of the femur but developed recurrence at the amputation site. The median time from surgery to local recurrence was 287.5 days (IQR, 250 to 315 days).

Lymph node metastases after limb amputation occurred in 2 cats $(2 / 66$ [3.0\%]; $95 \%$ CI, $0.2 \%$ to $11.0 \%)$. Superficial cervical lymph node metastasis diagnosed by excisional biopsy was observed 93 days after surgery in 1 cat with osteosarcoma of the proximal humerus, and inguinal lymph node metastasis was diagnosed by imaging in another cat with scapular osteosarcoma at 148 days after surgery.

The rate of distant metastasis after limb amputation was $41.9 \%(26 / 62 ; 95 \%$ CI, $30.5 \%$ to $54.3 \%)$, and the overall distant metastasis rate, including 5 cats with distant metastasis at the time of amputation, was $46.3 \%(31 / 67$; $95 \% \mathrm{CI}, 34.9 \%$ to $58.1 \%)$. The sites of metastases in the 26 cats with distant metastasis after amputation (with duplication) were lung ( $\mathrm{n}=19)$, bone $(\mathrm{n}=4)$, skin $(\mathrm{n}=2)$, subcutaneous $(n=2)$, and liver $(n=1)$. The median time from

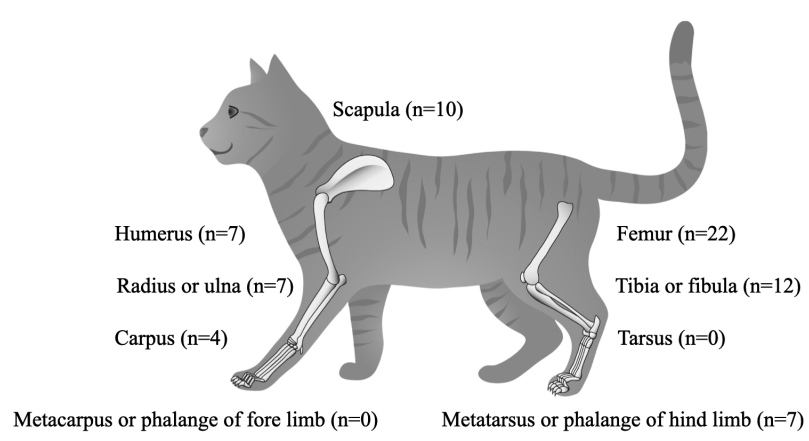

Figure I-Locations of osteosarcomas in cats. The femur was the most common tumor location. One cat is represented twice because lesions were found in the femur and tibia. 


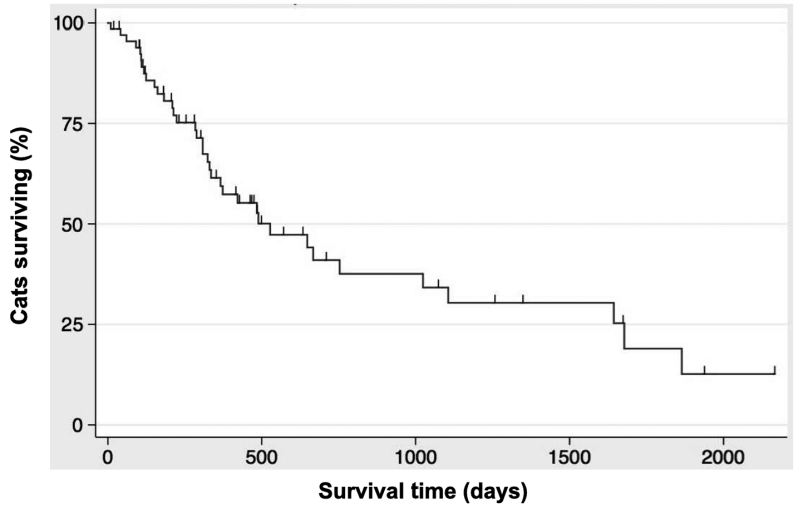

Figure 2-Kaplan-Meier survival curve for 67 cats with osteosarcoma. Survival time was calculated as the number of days from the date of limb amputation to death from any cause. Hash marks represent time of censoring.

surgery to metastasis in these 26 cats with metastasis after amputation was 235 days (IQR, 120 to 265 days).

Age was not associated with the presence of distant metastasis $(P=0.140)$. However, the presence of distant metastasis was significantly associated with tumor location and was significantly $(P=0.043)$ more common in cases with osteosarcoma of the humerus (humerus 6/7 [85.7\%] vs other 25/60 [41.7\%]). Osteosarcoma of the humerus was also associated with distant metastasis in multivariate analysis (adjusted OR, 9.56; $P=0.046$ ).

Regarding the outcomes at the time of analysis, 41 cats had died (41/67 [61.2\%]), 14 were still alive (14/67 [20.9\%]), and 12 were lost to follow-up (12/67 [17.9\%]). Of the 41 cats that died, 5 were censored at the last day of confirmed survival because the date of death was unknown. In addition, 6 of the 12 cats censored because they were lost to follow-up had distant metastases. Six of the 41 cats that died were determined by veterinarians to have died from causes other than osteosarcoma. The median overall survival time was 527 days (IQR, 284 to 1,678 days; Figure 2). Neither age nor humeral tumor location was associated with survival time in univariate or multivariate analyses.

\section{Discussion}

The present study revealed that distant metastasis occurred in approximately $40 \%$ of cats with appendicular or scapular osteosarcoma after limb amputation. In addition, osteosarcoma of the humerus had a high incidence of distant metastasis. To the best of our knowledge, this is the first study to investigate the rate of distant metastasis, survival time, and the associated risk factors in cats with appendicular or scapular osteosarcoma after amputation in the last 2 decades.

With the inclusion of 5 cases that had metastasis at the time of limb amputation, $46.3 \%$ of cats with appendicular or scapular osteosarcoma treated by limb amputation developed distant metastasis. In the present study, cases for which the presence or absence of distant metastasis was unknown based on the questionnaire survey were treated as having no distant metastasis to avoid overestimating the distant metastasis rate; however, if the unknown cases were excluded from the analysis, the distant metastasis rate was higher, at $55.4 \%$, indicating that the true incidence of distant metastases may be greater than 46.3\%. Detailed follow-up, improved imaging accuracy and diagnostic techniques, and the widespread availability of CT scans may account for the apparently higher rate of distant metastasis detected in this study, compared with previous studies. $3,5,6$

Cats with osteosarcoma of the humerus were more likely to develop distant metastases than cats with osteosarcomas at other sites. Similarly, a metaanalysis in dogs with osteosarcoma also reported that a proximal humeral location was a negative prognostic factor for survival time and disease-free interval, ${ }^{10}$ and osteosarcomas of the proximal humerus were associated with poorer metastasis-free survival in humans. ${ }^{11,12}$ Osteosarcomas in the proximal humerus may be associated with shorter survival and a shorter disease-free interval in dogs, compared with other sites, because they may be detected at a more advanced stage than tumors at other sites. ${ }^{10}$ The current results however indicated that, although a humeral location was associated with distant metastasis, it was not a negative prognostic factor for survival. This apparent discrepancy may be because 3 of the 7 cats with humeral osteosarcomas were censored in the survival analysis because the date of death was unknown, even though 3 of the cases were known to be dead at the time of the survey.

It is necessary to inform the cat's owners that distant metastasis may occur in approximately $40 \%$ of cases with appendicular or scapular osteosarcoma treated by limb amputation, with subsequent tumorrelated death. Follow-up postamputation imaging examinations are therefore recommended, and postoperative adjuvant chemotherapy should be considered.

This study had some limitations. It was a retrospective multicenter study, and there was therefore no standardized method for diagnosing metastasis and recurrence. In addition, detailed follow-up may not have been performed, because appendicular osteosarcoma has previously been considered to have a good prognosis following amputation alone, leading to assumed low risks of metastasis and recurrence. A second limitation was the lack of information and analysis of histopathologic factors, such as degree of cell differentiation, mitotic index, pleomorphism, and necrosis, as possible predictor variables in this study. Third, there was a possibility of reporting bias. Although we sampled all consecutive cases, only 2/3 of the cases were available for survival analysis. It is possible that facilities with worse outcomes, including metastasis, may have been more likely to answer the questionnaire, in which case the metastasis rate might have been overestimated. However, even if we assume that all 35 cases without questionnaires 
and medical records did not metastasize, the distant metastasis rate would have been $30.4 \%$ (31/102), which is still higher than previously reported rates. Further prospective studies are therefore needed, including physical examinations and imaging tests performed at defined intervals, to provide a more accurate diagnosis and follow up the prognosis to confirm metastasis and recurrence.

In conclusion, distant metastasis occurs in approximately $40 \%$ of cats with appendicular or scapular osteosarcoma after limb amputation. Furthermore, osteosarcoma of the humerus has the highest risk of distant metastasis. We therefore suggest that detailed follow-up is necessary, even after limb amputation, especially in cats with osteosarcoma of the humerus.

\section{Acknowledgments}

Funded by a Grant-in-Aid for Scientific Research (No. 25925026). Funding sources did not have any involvement in the study design, data analysis, and interpretation, or writing and publication of the manuscript.

The authors declare that there were no conflicts of interest.

Presented orally in part at the 10th Annual Conference of the Japanese College of Veterinary Internal Medicine, Yokohama, Japan, February 2014.

\section{References}

1. Dorn CR, Taylor DO, Schneider R, et al. Survey of animal neoplasms in Alameda and Contra Costa Counties, California. II. Cancer morbidity in dogs and cats from Alameda County. J Natl Cancer Inst. 1968;40(2):307-318.
2. Liu SK, Dorfman HD, Patnaik AK. Primary and secondary bone tumours in the cat. J Small Anim Pract. 1974;15(3):141156. doi:10.1111/j.1748-5827.1974.tb05671.x

3. Quigley PJ, Leedale AH. Tumors involving bone in the domestic cat: a review of fifty-eight cases. Vet Pathol. 1983;20(6):670-686. doi:10.1177/030098588302000603

4. Bitetto WV, Patnaik AK, Schrader SC, et al. Osteosarcoma in cats: 22 cases (1974-1984). J Am Vet Med Assoc. 1987; 190(1):91-93.

5. Dimopoulou M, Kirpensteijn J, Moens H, et al. Histologic prognosticators in feline osteosarcoma: a comparison with phenotypically similar canine osteosarcoma. Vet Surg. 2008;37(5):466-471. doi:10.1111/j.1532-950X.2008.00409.x

6. Kessler M, Tassani-Prell M, von Bomhard D, et al. Osteosarcoma in cats: epidemiological, clinical and radiological findings in 78 animals (1990-1995). Tierarztl Prax. 1997;25(3): 275-283.

7. Heldmann E, Anderson MA, Wagner-Mann C. Feline osteosarcoma: 145 cases (1990-1995). J Am Anim Hosp Assoc. 2000;36(6):518-521. doi:10.5326/15473317-36-6-518

8. Baum JI, Skinner OT, Boston SE. Fracture-associated osteosarcoma of the femur in a cat. Can Vet J. 2018;59(10):1096-1098.

9. von Elm E, Altman DG, Egger M, et al. Strengthening the reporting of observational studies in epidemiology (STROBE) statement: guidelines for reporting observational studies. BMJ. 2007;335(7624):806-808. doi:10.1136/ bmj.39335.541782.AD

10. Boerman I, Selvarajah GT, Nielen M, et al. Prognostic factors in canine appendicular osteosarcoma-a meta-analysis. BMC Vet Res. 2012;8:56. doi:10.1186/1746-6148-8-56

11. Cho WH, Song WS, Jeon DG, et al. Differential presentations, clinical courses, and survivals of osteosarcomas of the proximal humerus over other extremity locations. Ann Surg Oncol. 2010;17(3):702-708. doi:10.1245/s10434-009-0825-6

12. Song WS, Kong CB, Jeon DG, et al. Prognosis of extremity osteosarcoma in patients aged 40-60 years: a cohort/case controlled study at a single institute. Eur J Surg Oncol. 2010;36(5):483-488. doi:10.1016/j.ejso.2010.03.006 